\title{
Validación del cuestionario de Salud Eduactiva
}

\author{
José Miguel García Ramírez. Universidad de Granada \\ Irene Álvarez Castro. Universidad de Granada \\ Laura María Campos Rodríguez. Universidad de Granada \\ Tanía Fernández García. Universidad de Granada \\ Sofía Cassandra García McMullin. Carlenton University \\ Antonio Ibáñez Praena. Universidad de Granada \\ Marta López Márquez. Universidad de Granada \\ Alicia Ortega Castro. Universidad de Granada \\ Cristina Varón Povedano. Universidad de Granada
}

Recepción: 30 de junio de 2016 | Aceptado: 23 de febrero de 2017

Correspondencia: José Miguel García Ramírez | Correo-e: miguelgr@ugr.es

\author{
iD 0000-0002-9142-6503 \\ Citar: Garcia-Ramirez, JM, Alvarez, I, Campos, L, Fernandez, T, Ibañez, A, Lopez, M, Ortega, A \\ y Varon, C (2017). ReiDoCrea, 6, 64-73.
}

Nota: Este artículo muestra parte de los resultados obtenidos del Proyecto de Innovación Docente 14-90 de la Universidad de Granada "Salud Eduactiva: Riesgos Psicosociales y Salud Educacional. También ha sido publicado en la memoria de los PID de 2014-16 de la Universidad de Granada.

Resumen: En el ámbito universitario el estrés y el burnout, acompañados de una falta de actividad física y de una dieta alimenticia adecuada actúan contra la salud y bienestar psicológico de su alumnado. Este estudio adapta y valida un cuestionario para evaluar y prevenir el deterioro de la salud y el bienestar psicológico subjetivo del alumnado de la Universidad de Granada, a partir del cuestionario istas21 (Moncada, Llorens, Navarro y Kristensen, 2005) y la escala de flow (Jackson y Eklund, 2002). También se adapta y propone un modelo de intervención a partir del Modelo Circumplejo de las Emociones (Russel, 1978) y el Modelo Bidimensional del Bienestar Subjetivo en el Trabajo (Bakker y Oerlemans, 2011) para reducir el estrés y el burnout en estudiantes.

Palabras clave: Salud Educacional | Bienestar Psicológico

Validation of the Active Educational Health Questionnarie

Abstract: In the university context, stress and burnout, accompanied by a lack of physical activity and a proper diet are detrimental to the health and psychological well-being of the students. This study adapts and validates a questionnaire to evaluate and prevent the deterioration of the health and subjective psychological well-being of the students of the University of Granada, using the istas21 questionnaire (Moncada, Llorens, Navarro and Kristensen, 2005) and the flow scale (Jackson and Eklund, 2002). It also adapts and proposes an intervention model based on the Circumplex Model of Emotions (Russell, 1978) and the Two-Dimensional Model of Work-Related Subjective Well-Being (Bakker and Oerlemans, 2011) to reduce stress and burnout in students.

Keywords: Educational Health | Psychological Well-Being

\section{Introducción}

Los factores psicológicos, tanto positivos como negativos, juegan un papel trascendental en la salud, pues afectan al bienestar subjetivo, que es el grado en que una persona juzga de un modo general o global su vida en términos positivos (Veenhoven, 1984). Dentro del bienestar subjetivo se puede encontrar dos elementos: el balance afectivo y la satisfacción vital, percibida como estable y con un mayor componente cognitivo (Lucas, Diener y Suh, 1996). Estos dos elementos suponen diferentes marcos temporales del bienestar subjetivo, ya que la satisfacción vital es un 
juicio global de la propia vida, mientras que el balance afectivo hace referencia a la frecuencia relativa de afectos placenteros o displacenteros en la experiencia inmediata (Keyes, Shmotkin y Ryff, 2002). Este bienestar actúa como factor protector de la salud general, tanto mental como fisiológica (Vázquez, Hervás, Rahona y Gómez, 2009).

Aunque la salud percibida es totalmente subjetiva, una percepción positiva de salud viene determinada por un sentimiento positivo hacia uno mismo, una mayor sensación de control personal y una visión optimista de futuro. Asimismo, es precursora de habilidades de afrontamiento ante problemas cotidianos, que cobra especial importancia a la hora de resolver situaciones con un nivel de estrés elevado y que se perciben como amenazantes (Taylor, Kemeny, Reed, Bower y Gruenewald, 2000). Ese estado de bienestar facilita que las personas alcancen un mayor desarrollo psicológico, social y comunitario (Fredrickson, 2009).

También una dieta alimenticia adecuada y equilibrada ayuda a alcanzar un estado psicofisiológico optimo; por ejemplo, una dieta rica en triptófano mejora los niveles de serotonina, la cual es necesaria para regular el estado de ánimo (Alenina y Klempin, 2015); el triptófano, también, mejora los niveles de melatonina la cual ayuda a evitar o reducir los efectos de la depresión y/o la fatiga (Acuña-Castroviejo, 2006). Por lo tanto, una dieta alimenticia adecuada y equilibrada influye en el comportamiento (Dufour, 2010); también influye en la regulación entre el sistema neuroendocrino y las emociones positivas, así como en la regulación del sistema inmunológico (Mathew y Paulose, 2011). Dicha alimentación acompañada de actividad física mejora la salud general y mental, así como el estado de ánimo y la emotividad (Biddle, Fox y Boutcher, 2000; Cintra y Balboa, 2011), disminuye el nivel de ansiedad (Akandere y Tekin, 2004) y mejora el afrontamiento del estrés (Holmes, 1993). Las personas que comen alimentos saludables y practican ejercicio físico a diario reconocen tener una buena salud en general (Jiménez, Martínez, Miró y Sánchez, 2008).

Desde otro enfoque, cabe destacar la influencia de las emociones en la salud. Las emociones, que pueden ser duraderas o temporales, pueden tener muchas facetas que afectan al ser humano y son responsables del comportamiento, la percepción y las respuestas fisiológicas (Gross y Thompson, 2007). Dentro de las emociones se pueden distinguir dos dimensiones, el nivel de activación y la percepción que se tiene de ésta. A partir de ellas se genera el Modelo Circumplejo de las Emociones (Russel, 1978), en el que los estados emocionales se representan a través de dimensiones que dependen de cuatro niveles: alta o baja activación y agradable o desagradable.

En el día a día, las personas se encuentran en situaciones en las que es necesario regular las emociones para poder afrontar retos o problemas concretos. Regular las emociones supone modificar los procesos fisiológicos, de cognición y motivación para alcanzar logros. En este modelo el lado agradable viene orientado por actividades mentales, de comportamiento o motrices que regulan los estados afectivos y que al mismo tiempo aumentan o disminuyen la activación (Chamberlain, 1988; Mestre et al., 2012). De esta forma, las personas con una alta intensidad afectiva se situarán en la parte superior del modelo; es decir, viven con mucha intensidad cualquier momento, tanto agradable como desagradable. Sin embargo, el hecho de vivir con más intensidad no implica que las emociones sean positivas (García-Martín, 2002). El afecto negativo viene determinado por variables internas, como la salud percibida, mientras que el afecto positivo viene dado por elementos externos, como la alimentación y la actividad física (Bryant y Veroff, 1984).

Cuando las personas se encuentra en una situación en la que la activación es alta y desagradable aparece el estrés como reacción emocional, cognitiva, fisiológica y de 
comportamiento ante los aspectos adversos, lo que proporciona una percepción de incapacidad ante el hecho de no poder superar un acontecimiento concreto. Cuando esta situación se prolonga en el tiempo y pasa a una baja activación, entonces las personas afectadas tienen la sensación de estar quemado o Síndrome de Burnout, que es una combinación de cansancio emocional crónico, fatiga física, pérdida de interés, baja realización personal y deshumanización en el cuidado (Schaufeli y Enzmann, 1998). Este Síndrome de Burnout, a pesar de que es principalmente investigado en el ámbito profesional, también se ha analizado en estudiantes y se han presentado altos niveles de agotamiento así como de sentimientos de ineficacia, con actitud cínica y distante hacia el estudio (Gold y Michael, 1985; Powers y Gose, 1986).

Cuando la activación es baja y agradable se produce satisfacción y percepción de logro; la satisfacción y la felicidad pueden correlacionar pero no son equivalentes, sin embargo ambas influyen en la calidad de vida de cada persona (Campbell, Converse y Rodgers, 1976). En situaciones agradables y con una alta activación surge el estado de flow, el cual se caracteriza por: una alta concentración, sensación de control sobre la tarea, atención centrada exclusivamente en la tarea, distorsión del tiempo y sensación de vivir una experiencia autotélica (Csikszentmihalyi, 1997,1990 y 2000). Para alcanzar un estado de flow, han de darse tres situaciones: claridad de la metas, retroalimentación inmediata y equilibrio entre habilidades y desafíos respecto a la tarea. Cuando una persona se encuentra en estado de flow siente la necesidad de reexperimentar la sensación y de repetir la tarea favoreciendo el crecimiento personal, involucrándose en desafíos más complejos para satisfacer sus expectativas cada vez mayores (Csikszentmihalyi y Nakamura, 2002).

La experiencia de flow favorece la expansión de las metas, intereses y el crecimiento de habilidades entorno a su interés. Asimismo, tiene un impacto directo en el bienestar subjetivo de la persona, promoviendo emociones positivas (Moneta, 2004). Dichas emociones amplían el repertorio de actuación de las personas y favorecen la resiliencia y otros beneficios adaptativos (Fredrickson, 1998, 2001) que generan bienestar subjetivo.

\section{Objetivos}

- Validar una escala que mida la salud y satisfacción percibida por estudiantes universitarios.

- Proponer un Modelo de Salud y Bienestar Psicológico para alumnado universitario.

\section{Método}

\section{Participantes}

Participaron 1338 estudiantes, 465 hombres y 873 mujeres, de edades comprendidas entre 18 y $56(M=21,89 ; D T=3,856)$ y que estudiaban diferentes grados. Todos los datos están expuestos con más detalles en las siguientes tablas y gráfico:

\begin{tabular}{|lcc|}
\hline \multicolumn{3}{|c|}{ Tabla 1. Frecuencia de género } \\
\hline & Frecuencia & Porcentaje \\
\cline { 2 - 3 } Hombre & 465 & 34,8 \\
Mujer & 873 & 65,2 \\
Total & 1338 & 100,0 \\
\hline
\end{tabular}

\begin{tabular}{|c|c|c|c|}
\hline Mínimo & Máximo & Media & Desv. típ. \\
\hline 18 & 56 & 21,89 & 3,856 \\
\hline
\end{tabular}




\begin{tabular}{|lcc|ccc|}
\hline \multicolumn{7}{|c|}{ Tabla 3. Grados } \\
\hline Grados & Frecuencia & Porcentaje & Grados & Frecuencia & Porcentaje \\
\hline Arquitectura & 18 & 1,3 & Biología & 29 & 2,2 \\
Derecho & 18 & 1,3 & Educación Infantil & 132 & 9,9 \\
Educación Primaria & 444 & 33,2 & Educación Social & 83 & 6,2 \\
Enfermería & 19 & 1,4 & Farmacia & 31 & 2,3 \\
Filosofía & 16 & 1,2 & Ingeniería & 67 & 5,0 \\
Medicina & 47 & 3,5 & Odontología & 13 & 1,0 \\
Otros (menores de 1\%) & 45 & 3,4 & Psicología & 112 & 8,4 \\
Trabajo Social & 201 & 15,0 & Traducción e Interpretación & 23 & 1,7 \\
Máster & 40 & 3,0 & & & \\
\hline Total & 1338 & 100,0 & & & \\
\hline
\end{tabular}

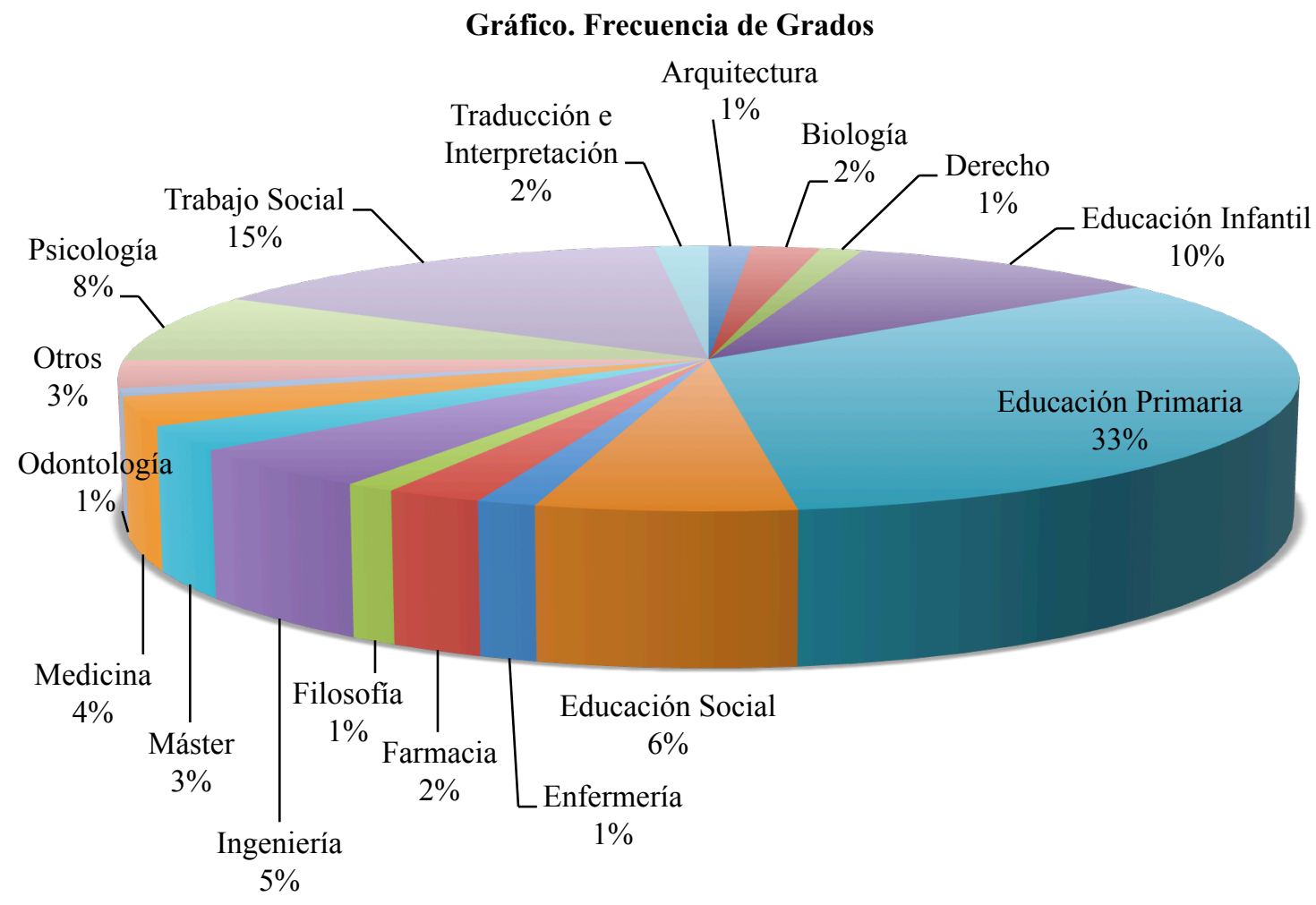

\section{Instrumento}

El instrumento está constituido por ítems correspondientes a las dimensiones de salud y satisfacción del cuestionario ISTAS21 (Moncada, Llorens, Navarro y Kristensen, 2005) recogidas en el anexo IV del Manual del método CoPsoQ-istas21 para la evaluación y riesgos psicosociales (Moncada, Llorens, Andrés, Moreno y Molinero, 2014), también se le ha añadido dos ítems de la escala de FLOW (Jackson y Eklund, 2002). El análisis de fiabilidad dio un Alfa de Cronbach de 0.915 (tabla 4).

\begin{tabular}{|cc|}
\hline Tabla 4. Estadísticos de fiabilidad \\
\hline Alfa de Cronbach & $\mathrm{N}$ de elementos \\
\hline, 915 & 17 \\
\hline
\end{tabular}




\section{Procedimiento}

El cuestionario se pasó aleatoriamente a alumnado de la Universidad de Granada durante el segundo semestre del curso académico 2015-16. Todo el alumnado participante fue informado del objetivo del instrumento y que era de carácter voluntario y anónimo. La recogida de datos se cerró en junio de 2016, para proceder a analizarlos.

\section{Análisis de datos}

Se realizó un análisis factorial de los datos obtenidos, a través del programa IBM SPSS Statistics 22.

\section{Resultados}

El análisis factorial tiene una medida de adecuación muestral de Kaiser-Meyer-Olkin alta $\left(\mathrm{KMO}_{136}=.918 ; \mathrm{x}^{2}=13065.034 ; \mathrm{p}=.00\right)$ y muestra 6 factores tal como se puede apreciar en la tabla 5. Por lo tanto, después de los resultados obtenidos del análisis estadístico se demuestra que el cuestionario tiene validez y fiabilidad en su aplicación a alumnado universitario. También se calculó el rango de riesgo aproximado al tanto por ciento redondeado.

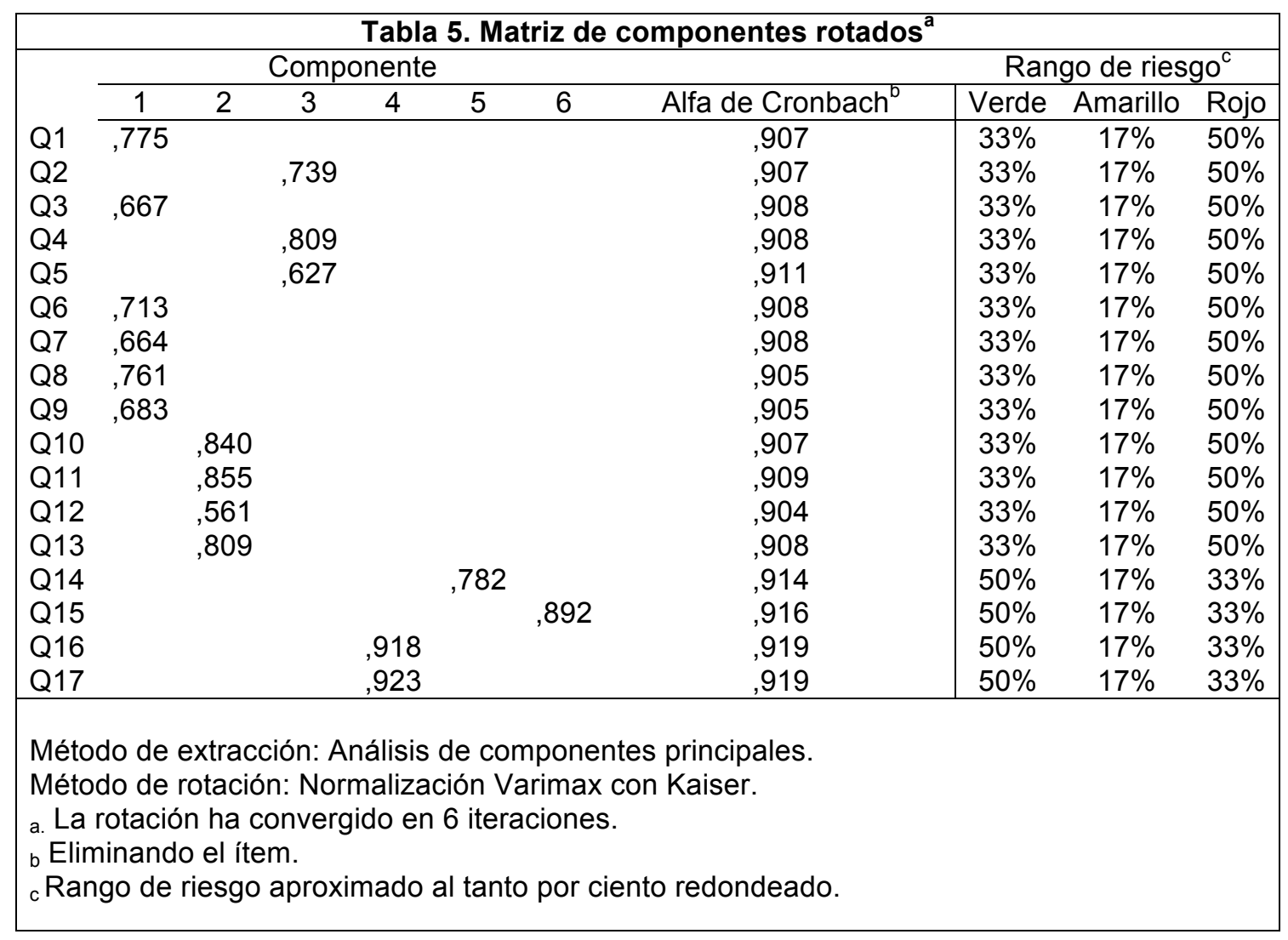


A continuación se expone los resultados del agrupamiento de los ítems por dimensiones:

- Salud Mental : Q2, Q4, Q5

- Estrés: Q1, Q3, Q6, Q7, Q8, Q9

- Burnout: Q10, Q11, Q12, Q13
- Salud General: Q14

- Satisfacción: Q15

- FLOW: Q16, Q17

El cuestionario, que está en el anexo, es de uso público y gratuito.

\section{Discusión}

La adaptación y validación de este instrumento es útil para recoger información sobre la salud general y satisfacción percibida por el alumnado, así como el flow; pero la clave está en desarrollar un modelo de intervención que ayude a comprender y mejorar el bienestar psicológico del alumnado.

Por lo tanto, este estudio también incluye la adaptación del Modelo Circumplejo de las Emociones (Russel, 1978) y el Modelo Bidimensional del Bienestar Subjetivo en el Trabajo (Bakker y Oerlemans, 2011). Ambos modelos representan las dimensiones relacionadas con los factores psicosociales, a través de dos ejes cartesianos estructurando los cuatro niveles explicados anteriormente: alta o baja activación y agradable o desagradable. A continuación, en siguiente gráfico 2 se puede apreciar la adaptación del modelo al que se ha llamado Modelo de Salud Eduactiva.

Gráfico 2. Modelo bidimensional de bienestar psicológico subjetivo para estudiantes universitarios, Modelo de Salud Eduactiva, el cual es una adaptación del Modelo Circumplejo de las Emociones (Russel, 1978) y Modelo Bidimensional del Bienestar Subjetivo en el Trabajo (Bakker y Oerlemans, 2011).

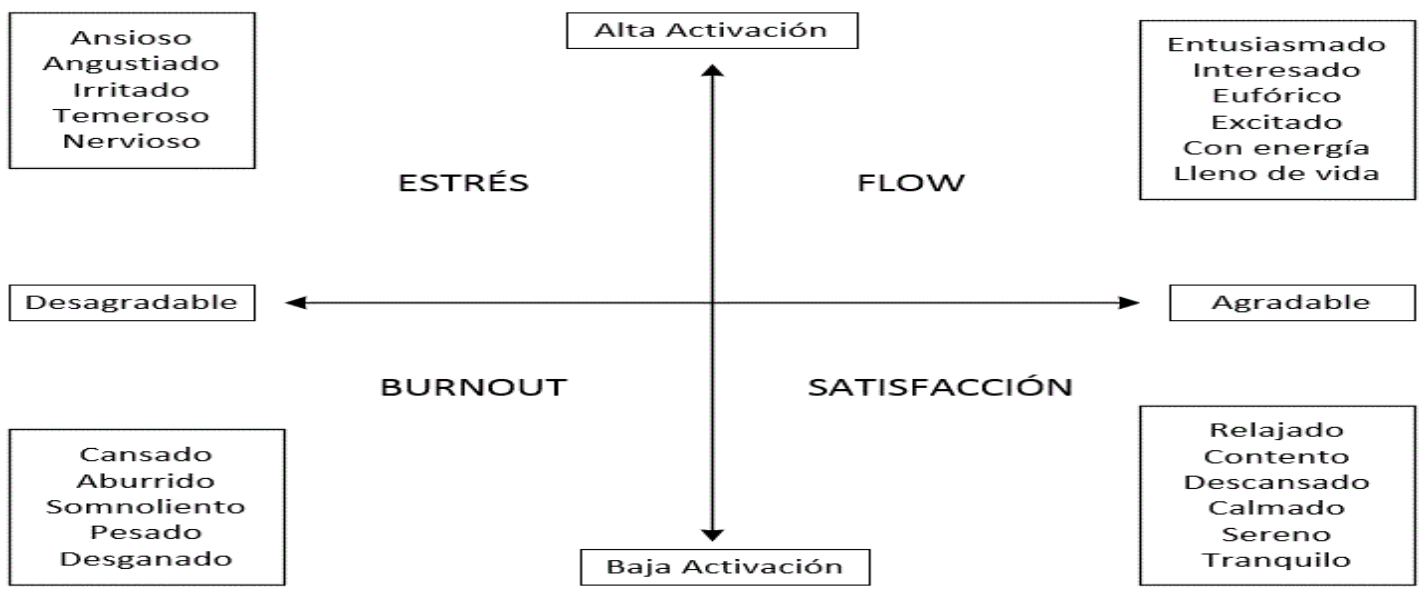

Este modelo bidimensional en el ámbito de la educación universitaria junto al cuestionario tienen como misión intervenir adecuadamente ante situaciones en las que los factores psicosociales afectan al alumnado. Además se complementa a través del control y seguimiento de la alimentación, así como de la promoción de la actividad física.

Para concluir, es necesario investigar, desarrollar e implementar modelos que ayuden a mejorar la salud y bienestar psicológico de las personas en cualquier ámbito en el que se encuentren. 


\section{Referencias}

ACUÑA-CASTROVIEJO, D: "Informe científico sobre el efecto de los campos electromagnéticos en el sistema endocrino humano y patologías asociadas". Grupo de Investigación: Comunicación Intercelular. Universidad de Granada (2006).

AKANDERE, M y TEKIN, A: "Efectos del Ejercicio Físico sobre la Ansiedad", PubliCE Standard (2004).

ALENINA, N, \& KLEMPIN, F: "The role of serotonin in adult hippocampal neurogenesis", Behavioural Brain Research, 277, 49-57 (2015).

BAKKER, AB, \& OERLEMANS, W: "Subjective well-being in organizations", The Oxford Handbook of Positive Organizational Scholarship, 178-189 (2011).

BIDDLE, SJH, FOX KR, \& BOUTCHER, SH: "Physical activity and psychological well-being", London: Routledge (2000).

BRYANT, FB, \& VEROFF, J: "Dimensions of subjective mental health in american men and women", Journal of Health and Social Behavior, 25, 116-135 (1984)

CAMPBELL, A, CONVERSE, PE, \& RODGERS, WL: "The Quality of American Life: Perceptions, Evaluations and Satisfaction". New York: Russell (1976).

CHAMBERLAIN, K: "On the Structure of Subjective Well-Being", Springer, 20(6), 581-604 (1988).

CINTRA, O y BALBOA, Y: "La actividad física: un aporte para la salud”, Educación física y deportes, 159, 21-26 (2011).

CSIKSZENTMIHALYI, M, \& NAKAMURA, J: "The Concept of Flow", in CR Snyder, \& SJ López (Eds.), "Handbook of Positive Psychology" (pp. 89-92), New York: Oxford University Press (2002).

CSIKSZENTMIHALYI, M: "Finding flow: The psychology of engagement with everyday life", Nueva York: Basic Books (1997).

CSIKSZENTMIHALYI, M: "Flow: the psychology of optimal experience”, Nueva York: Harper y Row, (1990).

CSIKSZENTMIHALYI, M: "The contribution of flow to positive psychology", in JE Gilham (Eds.), "The science of optimism and hope: research essays in honor of Martin P. Seligman" (pp. 387-395). Philadelphia: Templeton Foundation Press (2000).

DUFOUR, BD: "Nutritional up-regulation of serotonin paradoxically induces compulsive behaviour", Nutritional Neuroscience, 13(6), 256-264 (2010).

FREDRICKSON, BL: "Positivity”, NewYork: Crown (2009).

FREDRICKSON, BL: "The role of positive emotions in positive psychology: The broaden-and-build theory of positive emotions". American Psychologist: Special Issue, 56, 218-226 (2001).
FREDRICKSON, BL: "What good are positive emotions?" Review of General Psychology: Special Issue: New Directions in Research on Emotion, 2, 300-319 (1998).

GARCÍA-MARTÍN, MA: "El bienestar subjetivo", Escritos de Psicología, 6, 18-39 (2002).

GOLD, Y, \& MICHAEL, WB: "Academic self-concept correlates of potential burnout in a sample of first-semester elementary school practice teachers: A con-current validity study", Educational and Psychological Measurement, 45, 909-914 (1985).

GROSS, JJ, \& THOMPSON, RA: "Emotion Regulation: Conceptual foundations", In J.J. Gross, (Eds.), "Handbook of Emotion Regulation" (pp. 3-24), New York: Guilford (2007).

HOLMES, DS: "Aerobic fitness and the response to psychological stress", In P. Seraganian (Ed.), Exercise psychology. The influence on physical exercise on psychological process (pp. 39-63). Nueva York: John Wiley (1993).

JACKSON, SA, \& EKLUND, RC: "Assessing flow in physical activity: the flow state scale-2 and dispositional flow scale-2", Journal of Sport \& Exercise Psychology, 24(2), 133-150, (2002).

JIMÉNEZ, MG, MARTíNEZ, P, MIRÓ, E y SÁNCHEZ, Al: "Bienestar psicológico y hábitos saludables: ¿están asociados a la práctica de ejercicio físico?", International Journal of Clinical and Health Psychology. 8(1), 185-202 (2008).

KEYES, CLM, SHMOTKIN, D, \& RYFF, CD: "Optimizing wellbeing: the empirical encounter of two traditions", Journal of Personality and Social Psychology, 82, 1007-1022 (2002).

LUCAS, RE, DIENER, E, \& SUH, EM: "Discriminant validity of wellbeing measures", Journal of Personality and Social Psychology, 71, 616-628 (1996).

MATHEW, J, \& PALOUSE, CS: "The healing power of well-being", Acta Neuropsychiatrica, 23 (4), 145-155 (2011).

MESTRE, JM, GUIL, R, CORTIJO, M, RUH, N, SERRANO, N y JIMÉNEZ, M: "Regulación de emociones: una visión pragmática e integradora desde el modelo circumplejo", en Gonzalez, C., Gonzalez, D., Guil, R. y Mestre, J.M. (Eds.), "Aportaciones recientes al estudio de la motivación y de las emociones" (pp. 261-268), Sevilla: Fénix Editora (2012).

MONCADA, S, LLORENS, C, ANDRÉS, R, MORENO, N y MOLINERO, E: "Manual del método CoPsoQ-istas21 (versión 2) para la evaluación y la prevención de los riesgos psicosociales en empresas con 25 o más trabajadores y trabajadoras VERSIÓN MEDIA", Barcelona: Instituto Sindical de Trabajo, Ambiente y Salud (2014).

MONCADA, S, LLORENS, C, NAVARRO, A, KRISTENSES, TS: "ISTAS21: Versión en lengua castellana del cuestionario psicosocial de Copenhague (COPSOQ)", Archivos de Prevención de Riesgos Laborales, 8(1), 18-29 (2005). 
MONETA, GB: "The flow experience across cultures", Journal of Happiness Studies, 5, 115-121 (2004).

POWERS, S, \& GOSE, KF: "Reliability and construct validity of the Maslach Burnout Inventory in a sample of university students" Educational and Psychological Measurement, 46, 251-257 (1986).

RUSSELL, JA: "Evidence of convergent validity on the dimensions of affect", Journal of Personality and Social Psychology, 36, $1152-1168$ (1978).

SCHAUFELI, WB, \& ENZMANN, D: "The burnout companion to study and practice: a critical analysis", London: Taylor \& Francis (1998).

TAYLOR, SE, KEMENY, ME, REED, GM, BOWER, JE, \& GRUENEWALD, TL: "Psychological resources, positive illusions, and health", American Psychologist, 55(1), 99-109 (2000).

VÁZQUEZ, C, HERVÁS, G, RAHONA, JJ y GÓMEZ, D: "Bienestar psicológico y salud: Aportaciones desde la Psicología Positiva", Anuario de Psicología Clínica y de la Salud, 5, 15-28 (2009).
VEENHOVEN, R: "Conditions of Happiness", Dordrecht: Reidel (1984).

\section{Revisión por pares abierta | Open Peer Review}

Dra. Karen McMullin. Trent University (Canada)

iD 0000-0002-1449-3550

Dr. Alfonso Conde Lacarcel. Universidad de Granada (Spain)

iD $0000-0002-2247-2856$

Dr. Walfredo González Hernández. Universidad de Matanzas (Cuba)

iD $0000-0003-4028-4266$

\section{Anexo.}

Cuestionario de Salud Eduactiva: Salud, Satisfacción y Flow en estudiantes universitarios. 


\section{CUESTIONARIO DE SALUD EDUACTIVA: Salud, Satisfacción y Flow en estudiantes universitarios}

La respuesta a este cuestionario servirá para evaluar tu salud y bienestar psicológico. Para ello, es necesario que contestes TODAS las preguntas. Se trata de un cuestionario ANÓNIMO, de respuesta VOLUNTARIA e INDIVIDUAL. Por eso te pedimos que respondas sinceramente cada una de las preguntas, sin debatir con nadie. Las preguntas tienen diversas opciones de respuesta y tienes que señalar con un circulo la respuesta que consideres que describe mejor tu situación.

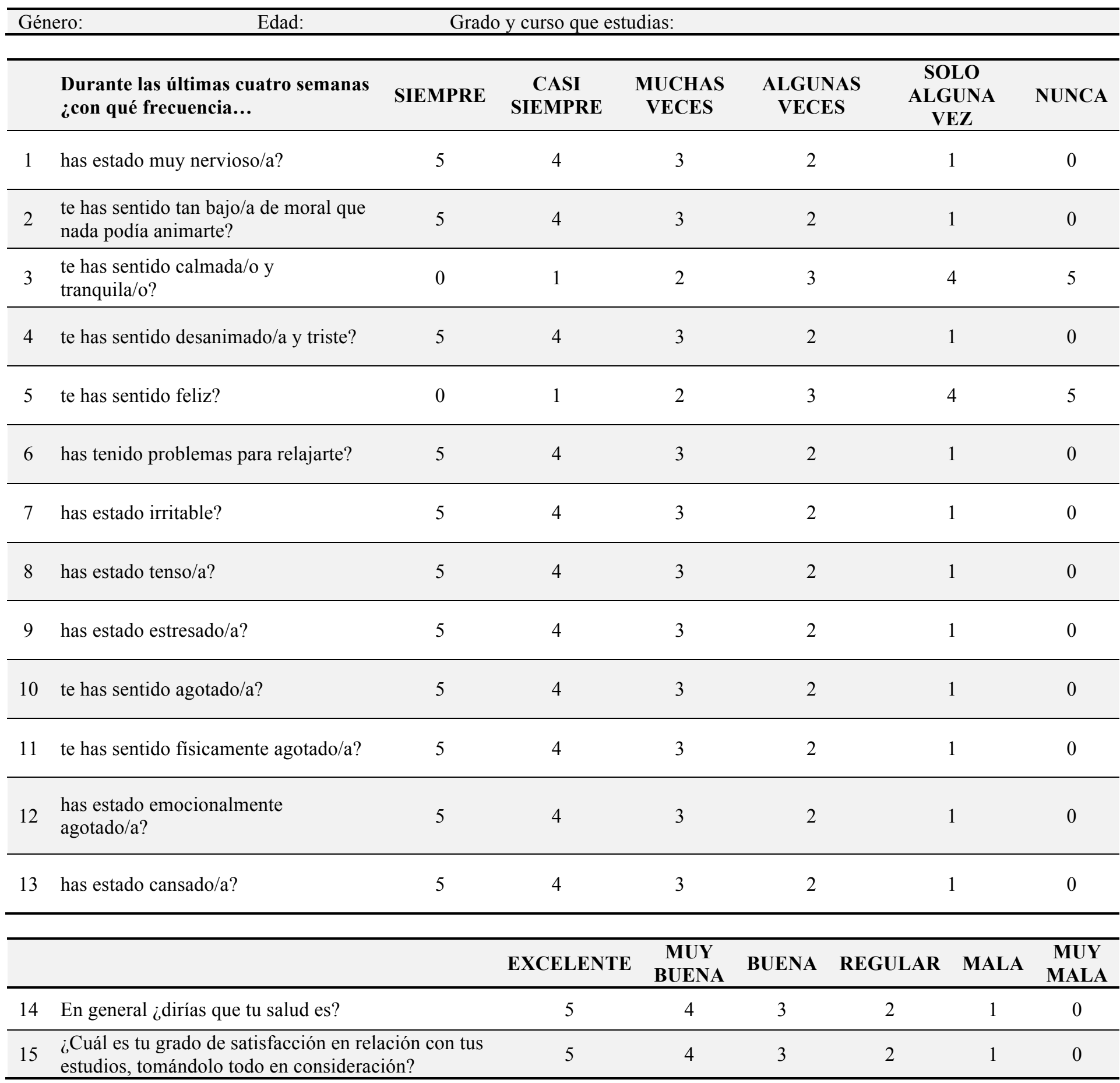

FLOW: es el estado mental cuando persona se encuentra totalmente inmersa y plenamente concentrado en lo que está haciendo o realizando. Por favor, indica con qué frecuencia has experimentado las siguientes frases.

\begin{tabular}{|c|c|c|c|c|c|c|c|}
\hline & & NUNCA & $\begin{array}{c}\text { CASI } \\
\text { NUNCA }\end{array}$ & $\begin{array}{c}\text { A } \\
\text { VECES }\end{array}$ & $\begin{array}{c}\text { A } \\
\text { MENUDO }\end{array}$ & $\begin{array}{c}\text { CASI } \\
\text { SIEMPRE }\end{array}$ & SIEMPRE \\
\hline 16 & $\begin{array}{l}\text { ¿En qué medida crees que en tu estudio diario } \\
\text { experimentas un estado de FLOW? }\end{array}$ & 0 & 1 & 2 & 3 & 4 & 5 \\
\hline
\end{tabular}




\section{ANALIZA TUS RESULTADOS}

Este cuestionario tiene por objetivo evaluar tu salud y bienestar psicológico. Los resultados obtenidos te servirán para conocer tu estado de salud y bienestar psicológico subjetivo, para posteriormente tomar medidas preventivas con la participación de tus compañeros/as y profesorado.

Con el fin de que puedas conocer tus resultados particulares sigue las siguientes indicaciones:

- Anota las puntuaciones obtenidas en la dimensión que corresponda y súmalas. Después buscas en que rango de riesgo se encuentra en las columnas de puntuaciones de referencia.

- Las puntuaciones dentro de la columna verde significa que estás en una situación favorable para tu salud y bienestar psicológico.

- Las puntuaciones dentro de la columna amarilla significa que estás en una situación intermedia y tendrías que preocuparte por tu salud y bienestar psicológico.

- Las puntuaciones dentro de la columna roja significa que estás en una situación desfavorable y tienes que tomar medidas para mejorar tu salud y bienestar psicológico.

DIMENSIONES

Suma las puntuaciones obtenidas de los ítems 2,4

SALUD

MENTAL y 5

$\longleftarrow^{+} \ldots^{+}{ }^{\prime}=$

Suma las puntuaciones obtenidas de los ítems 1,3 , ESTRÉS $6,7,8$ y 9

$\ldots^{+} \ldots^{+} \ldots^{+} \ldots^{+} \ldots^{+}{ }^{+}=$

Suma las puntuaciones obtenidas de los ítems 10 ,

BURNOUT 11,12 y 13

0-4

5-8

0-4

Pon la puntuación obtenida del ítem 15

\section{SATISFACCIÓN}

Pon la puntuación obtenida del ítem 14

SALUD
GENERAL

Suma las puntuaciones obtenidas de los ítems 16 y

FLOW

17

5-10

\section{RANGO DE RIESGO}

$\begin{gathered}\text { Situación } \\ \text { más } \\ \text { favorable }\end{gathered}$
$0-3$
$0-6$

4-6

7-15

Situación

más

intermedia

desfavorable

7-12

13-30 